\title{
Polysomnographic findings of obstructive sleep apnea in children with adenotonsillar hypertrophy
}

\author{
Érico Campos Moreira Silva ${ }^{1 \# \dagger}$, Vinícius S. Moraes ${ }^{1 \#}$, Helena Protetti ${ }^{2 \#}$, Silke T. Weber ${ }^{1 \#}$ \\ ${ }^{1}$ Ophtalmology, Otorhinolaryngology and Head \& Neck Surgery Department, Botucatu School of Medicine, São Paulo State \\ University, Botucatu, Brazil; ${ }^{\dagger}$ Corresponding Author: ericovcms@hotmail.com \\ ${ }^{2}$ Neurology, Neuro Clinics, Botucatu, Brazil
}

Received 10 June 2013; revised 10 July 2013; accepted 1 August 2013

Copyright (C) 2013 Érico Campos Moreira Silva et al. This is an open access article distributed under the Creative Commons Attribution License, which permits unrestricted use, distribution, and reproduction in any medium, provided the original work is properly cited.

\section{ABSTRACT}

Serous Background: There are few studies assessing the clinical manifestations of sleep breathing disorders and polysomnograms in several pediatric age ranges. This studied aimed to assess polysomnography results such as apnea-hypopnea index, mean oxygen saturation and sleep efficiency in children presenting with airway obstruction and adenotonsillar hypertrophy complaints, and to establish whether they are correlated to age and sex. Methods: A retrospective study with children of both sexes, aged between 2 and 12 years, with clinically suspected obstructive sleep apnea syndrome and adenotonsillar hypertrophy, who underwent polysomnography before surgery. The children were allocated to groups according to their age range (Group I: 2 to 4 years old; Group II: 5 to 8 years old; Group III: 9 to 12 years old). Apnea-hypopnea index, mean oxygen saturation and sleep efficiency data were compared between sexes and among the three groups (Student's $t$ test, $p<0.05)$. Results: Of 167 children studied by polysomnography, $76.6 \%$ were of school age and $67 \%$ were male. For all studied age ranges, there was no difference between sexes for the investigated parameters (body mass index, apnea-hypopnea index, mean oxygen saturation and sleep efficiency). As regards mean oxygen saturation, Group I showed the lowest value (89.9 \pm 6.2$)$. Apnea-hypopnea in-

\footnotetext{
"Competing interests: There is no financial relationship relevant to this article to disclose. There is no conflict of interest to this article to disclose.

${ }^{\#}$ These authors contributed equally to this work.
}

dexes were higher in male children aged between 2 and 4 years $(9.9 \pm 5.2)$. Group III had the lowest sleep efficiency $(84.1 \pm 9.2)$. Conclusion: There was a predilection of more severe cases of obstructive sleep apnea syndrome for children younger than four years, shown by higher apnea-hypopnea index per hour and lower mean oxygen saturation in this age range.

Keywords: Polysomnogram; Sleep Architecture; Children; Obstructive Sleep Apnea; Sleep

Disordered Breathing

\section{BACKGROUND}

Obstructive sleep apnea syndrome (OSAS) in children was first described by William Osler in 1892, but OSAS study only became systematic from 1976, after the publication of a study by Guilleminault et al. [1], who carried out polysomnography in children with snoring and mouth breathing. According to the "American Thoracic Society", OSAS in children is defined as "prolonged partial upper-airway obstruction and/or intermittent complete obstruction that disrupts normal ventilation during sleep and normal sleep patterns" [2]. Based on the International Classification of Sleep Disorders, OSAS is an "intrinsic sleep disorder characterized by repetitive episodes of upper-airway obstruction associated with hemoglobin desaturation" [3].

Prevalence of OSAS among children has varied from $0.7 \%$ to $3 \%$ in different epidemiological studies [3-5]. The incidence peaks in pre-school children, an age range in which palatine tonsil and adenoid hypertrophy are more common [6].

Children may have several types of breathing disorders during sleep: primary snoring, periodic breathing, 
upper-airway resistance syndrome and apnea-hypopnea (central, obstructive or mixed) [7].

There are few studies assessing the clinical manifestations of sleep breathing disorders and polysomnograms for several pediatric age ranges. Studies assessing possible differences between sexes are also scarce.

Our hypothesis is that younger children, whose airways are smaller and possibly more susceptible to collapse, due to upper-airway obstruction and adenotonsillar hypertrophy, would exhibit larger AHI values and lower oxygen saturation than older children during sleep assessed by polysomnography.

The objective of the present study was to assess the polysomnography results such as apnea-hypopnea index (AHI), mean oxygen saturation $\left(\mathrm{mSaO}_{2}\right)$ and sleep efficiency (SE) in children, as well as their correlation to age and sex.

\section{METHODS}

The study protocol was approved by the Research Ethics Committee of Botucatu Medical School, São Paulo, Brazil. The parents or guardians received explanations about the study and signed the Free and Informed Consent Form.

This retrospective and descriptive study involved children, aged between 2 and 12 years with adenotonsillar hypertrophy, of both sexes, who underwent polysomnography at Botucatu Neuroclinics, São Paulo, Brazil, between January 2008 and December 2011. All children were referred to the Neuroclinics Service by pediatricians or otorhinolaryngologists for assessment of OSAS diagnosis and possible indication for tonsil surgery. The criteria for suspected OSAS in children were: recurrent snoring, breathing pauses mentioned by parents or guardians and restless sleep.

The selection followed the inclusion and exclusion criteria cited in Table $\mathbf{1}$.

Overnight polysomnography was carried out in computerized equipment Alice 4, Respironics, with minimal duration of 5 hours, in a quiet environment, and the child was accompanied by one of his/her parents or guardians. The reports were issued by one single physician, authorized by the Brazilian Sleep Association. The following data were recorded: electroencephalography (EEG), submental electromyography, tibialis anterior muscle electromyography, electrooculography, electrocardiography (ECG), oronasal airflow, thoracoabdominal movements (breathing effort), pulse oximetry, snoring intensity and changes in position along the night, as recommended [8].

For our study, the following polysomnography data were analyzed:

AHI-apnea-hypopnea index: number of apneas and hypopneas per slept hour. Snoring: presence or not.
Table 1. Inclusion and exclusion criteria for the studied population.

\begin{tabular}{|c|c|}
\hline Inclusion Criteria & Exclusion criteria \\
\hline Both sexes & Genetic Syndromes \\
\hline $\begin{array}{l}\text { Clinically suspected OSAS: snoring, } \\
\text { prolonged nocturnal breathing pauses, } \\
\text { restless sleep }\end{array}$ & Neurological diseases \\
\hline $\begin{array}{c}\text { Age } 2 \text { - } 12 \text { years } \\
\text { Adenotonsillar hypertrophy } \\
(\text { obstruction } \geq 75 \%)\end{array}$ & $\begin{array}{c}\text { Children using neuroleptics or } \\
\text { sedatives }\end{array}$ \\
\hline
\end{tabular}

$\mathrm{mSaO}_{2}$ - minimal, maximal and mean.

SE-Sleep efficiency.

Central apnea was considered the absence of breathing thoracoabdominal movement and flow for at least 10 seconds. Obstructive apnea was considered the absence of breathing flow but presence of thoracoabdominal movement for a period longer than 2 breathing cycles, and hypopnea was considered the reduction by $20 \%$ to $50 \%$ in the breathing flow associated with a fall in oxygen saturation by $3 \%$ or awakening, meeting the criteria suggested by the American Academy of Sleep Medicine (AASM, 2007) [9]. Apnea-hypopnea index (AHI): sum of the number of obstructive and mixed apneas, obstructive and mixed hypopneas, expressed as events per hour (considering for calculation the total time of sleep). For children, AHI $<1$ /hour is considered normal.

Oxygen saturation inferior to $92 \%$ was considered abnormal. Sleep efficiency superior to $89 \%$ was considered normal.

The records for each child were used to obtain the referral reason (suspected OSAS) and the related clinical data: age, sex, weight, height, BMI.

The children were allocated to three groups according to their age range, as follows:

Group I: children, both sexes, from 2 to 4 years old.

Group II: children, both sexes, from 5 to 8 years old.

Group III: children, both sexes, from 9 to 12 years old.

Polysomnograms were compared among groups. The data were compared for each age group and sex (Student's t test, for independent samples) and correlated to age (Spearman's test).

\section{RESULTS}

Of the total 253 polysomnography tests carried out for children in the age range between two and 12 years in this period, 167 children met the inclusion and exclusion criteria (Table 1). Of these, $128(76.6 \%)$ were of school age and $111(67 \%)$ were male (Figure 1).

There were no significant differences between sexes in all age ranges for all studied variables: BMI, AHI, $\mathrm{mSaO}_{2}$, SE (Table 2). As to mean oxygen saturation, the 
group of children aged between 2 and 4 years showed the lowest value (Table 2, $\mathrm{p}<0.05$ ).

Comparison of polysomnograms among the three age ranges of both sexes (Table 3) indicated that apnea-hypopnea index was higher in children of Group I $(\mathrm{p}<$ $0.05)$, mean oxyhemoglobin saturation was lower in children of Group I ( $p<0.05)$, whereas sleep efficiency was reduced in children of Group III $(p<0.05)$.

\section{DISCUSSION}

In the history of a child with OSAS, we frequently find: nocturnal snoring, breathing pauses, breathing difficulty, restless sleep, night sweats and mouth breathing [10]. We may also find: nocturnal enuresis, habit of sleeping in cervical hyperextension, excessive daytime sleepiness, behavioral changes, learning deficit and high blood pressure [11]. Nocturnal snoring is common both in cases of primary snoring and in OSAS and upper airway resistance syndrome, and the noise intensity may not be related to the apnea severity [5,7].

Polysomnography (PSG) in a sleep laboratory during a whole night is the gold standard for OSAS diagnosis. The test has excellent reproducibility [12], documents upper-airway obstruction, distinguishes obstructive from central apnea and records epileptic crises in children with neurological diseases [13]. In the literature, the criteria used for OSAS diagnosis in children vary [14-17]. On the other hand, the most accepted recommendations are those of AASM 2007 "American Thoracic Society" [9].

The classification of OSAS is also suggested as mild when $1 \leq \mathrm{AHI}<5$ /hour, moderate when $5 \leq \mathrm{AHI}<$

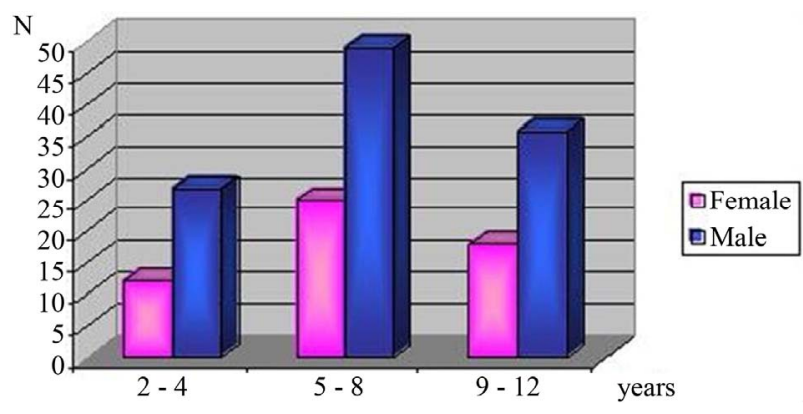

Figure 1. Distribution according to sex and age. 9/hour and severe when AHI > 10/hour [12].

In our study, most children referred to OSAS investigation were of school age, which coincides with the age of highest prevalence of adenotonsillar hypertrophy, as previously described in several studies $[3,4]$. In addition, a larger number of male children were referred, even though in this study there was no difference in polysomnograms between sexes in all age ranges, differently from what is described for adults. The difference in OSAS between sexes for adults (incidence and clinical manifestations) is suggested to have as factor, among others, anatomical variations such as height of the hyoid implantation or increased adipose tissue in the neck; most of them are mediated by the male hormone, which explains the absence of these differences in the studied age range [18].

Although polysomnography in children has become an increasingly common routine test, its reference values are not fully standardized yet, with several definitions for OSAS. We considered the definition most used in our reference (AASM, ATS) [2,9] and a severity classification suggested by Katz [12]. Thus, we observed that children of Group I (aged between 2 and 4 years) showed OSAS signs considered moderate, while in Groups II and III these signs were mild. The lowest levels of mean oxygen saturation and the highest apnea-hypopnea indexes were found for younger children $(\mathrm{p}<0.05)$, which may be related to a smaller airway diameter, as suggested in a previous study [19].

In contrast, sleep efficiency was significantly lower in Group III, for which a higher sleep fragmentation was expected with a larger number of obstructive events. We believe that in young children the electroencephalographic response of superficialization/awakening does not correspond to the classical plotting, standardized for adults; thus, awakening and microawakening in younger children may be under diagnosed, as already observed [20]. Another possible explanation for lower sleep efficiency values in older children is the influence of environmental factors, such as application of the test during school periods [21].

There are still controversies in literature about the criteria for PSG interpretation in children and teenagers.

Table 2. Comparison of means and standard deviation of BMI, $\mathrm{AHI}, \mathrm{mSaO}_{2}$ and $\mathrm{SE}$ between sexes for the different age ranges.

\begin{tabular}{ccccccccc}
\hline Age (years old) & \multicolumn{2}{c}{ BMI $\left(\mathrm{Kg} / \mathrm{m}^{2}\right)$} & \multicolumn{2}{c}{$\mathrm{AHI}(\mathrm{n} / \mathrm{h})$} & \multicolumn{2}{c}{$\mathrm{mSaO}_{2}$} & & SE (\%) \\
\hline & Female & Male & Female & Male & Female & Male & Female & Male \\
$2-4$ & $16.3 \pm 2.5$ & $18.3 \pm 6.2$ & $9.05 \pm 4.28$ & $9.75 \pm 7.62$ & $90.2 \pm 4.4$ & $89.4 \pm 9.9$ & $89.5 \pm 6.8$ & $83.5 \pm 13.9$ \\
$5-8$ & $17.2 \pm 3.7$ & $17.9 \pm 3.6$ & $4.9 \pm 3.3$ & $6.24 \pm 4.8$ & $94.3 \pm 3.1$ & $93.7 \pm 3.1$ & $86.2 \pm 11.3$ & $84.6 \pm 9.2$ \\
$9-12$ & $18.7 \pm 3.2$ & $18.5 \pm 2.9$ & $4.2 \pm 4.9$ & $5.4 \pm 3.6$ & $96.4 \pm 2.3$ & $95.8 \pm 3.4$ & $84.1 \pm 8.7$ & $84.1 \pm 10.8$ \\
\hline
\end{tabular}

BMI: body mass index AHI: apnea-hypopnea index. $\mathrm{mSaO}_{2}$ : mean oxyhemoglobin saturation. SE: sleep efficiency. 
Table 3. Comparison of means and standard deviation of AHI, $\mathrm{mSaO}_{2}$ and $\mathrm{SE}$ among the different age ranges.

\begin{tabular}{cccc}
\hline $\begin{array}{c}\text { Age } \\
\text { (years old) }\end{array}$ & $\mathrm{AIH}$ & $\mathrm{mSaO}_{2}$ & $\mathrm{SE}$ \\
\hline $2-4$ & $9.9 \pm 5.2^{*} \mathrm{a}$ & $89.9 \pm 6.2^{*} \mathrm{~b}$ & $87.8 \pm 10.7^{*} \mathrm{c}$ \\
$5-8$ & $5.2 \pm 3.6 \mathrm{~b}$ & $93.9 \pm 3.1 \mathrm{~b}$ & $86.1 \pm 9.5 \mathrm{~b}$ \\
$9-12$ & $4.8 \pm 3.9 \mathrm{~b}$ & $95.2 \pm 3.1 \mathrm{~b}$ & $84.1 \pm 9.2 \mathrm{~b}$ \\
\hline
\end{tabular}

*different letters represent statistical difference at $\mathrm{p}<0.05$.

As the duration and the characteristics of sleep/vigil cycle change over the individual's maturation, studies including large cohort of healthy children of different age ranges are needed to obtain normal PSG standards (ATS) [2].

Our study had the limitation of only including children with adenotonsillar hypetrophy and already showing clinical implications of OSAS and referred to polysomnography. Thus, there may have been selection in the population of younger children with more exuberant symptoms and more severe clinical signs, since for these children indication for surgery implies higher comorbidity.

\section{CONCLUSION}

Polysomnography variables do not seem to be different for sexes in the studied population. However, this study suggests a predilection of more severe OSAS signs for children younger than four years old, shown by the higher apnea-hypopnea index per hour and the lower mean oxygen saturation in this age range.

\section{AUTHORS' CONTRIBUTIONS}

ECMS: participated in the study design, clinical evaluation of the patients and draft of the manuscript;

VSM: participated with the clinical evaluation of the patients;

HP: participated in polysomnography analysis, statistical analysis;

STW: participated in the study design, coordination and draft of manuscript;

All authors read and approved the final manuscript.

\section{REFERENCES}

[1] Guilleminault, C., Eldridge, F.L., Simmons, F.B. and Dement, W.C. (1976) Sleep apnea in eight children. Pediatrics, 58, 23-30.

[2] American Thoracic Society (1996) Standards and indications for cardiopulmonary sleep studies in children. American Journal of Respiratory and Critical Care Medicine, 153, 866-878. doi:10.1164/ajrccm.153.2.8564147
[3] Brunetti, L., Rana, S., Lospalluti, M.L., Pietrafesa, A., Francavilla, R., Fanelli, M., et al. (2001) Prevalence of obstructive sleep apnea in a cohort of 1207 children of Southern Italy. Chest, 120, 1930-1935. doi:10.1378/chest.120.6.1930

[4] Anuntaseree, W., Rookkapan, K., Kuasirikul, S. and Thingsuksai, P. (2001) Snoring and obstructive sleep apnea in Thai school-age children: Prevalence and predisposing factors. Pediatric Pulmonology, 32, 322-327. doi:10.1002/ppul.1112

[5] Gislason, T. and Benediktsdóttir, B. (1995) Snoring, apneic episodes, and nocturnal hypoxemia among children 6 months to 6 years old. Chest, 107, 963-966. doi:10.1378/chest.107.4.963

[6] Bower, C. and Buckmiller, L. (2001) What's new in pediatric obstructive sleep apnea. Current Opinion in Otolaryngology \& Head and Neck Surgery, 9, 352-358. doi:10.1097/00020840-200112000-00003

[7] Anstead, M. (2000) Pediatric sleep disorders: New developments and evolving understanding. Current Opinion in Pulmonary Medicine, 6, 501-506. doi:10.1097/00063198-200011000-00007

[8] Masood, A. and Phillips, B. (2000) Sleep apnea. Current Opinion in Pulmonary Medicine, 6, 479-484. doi:10.1097/00063198-200011000-00003

[9] Sack, R.L., Auckley, D., Auger, R.R., et al. (2007) Circadian rhythm sleep disorders: Part II, advanced sleep phase disorder, delayed sleep phase disorder, free-running disorder, and irregular sleep-wake rhythm. An American Academy of Sleep Medicine review. Sleep, 30, 1484-1501.

[10] Çinar, U., Vural, C., Çakir, B., Topuz, E., Karaman, M.I. and Turgut, S. (2001) Nocturnal enuresis and upper-airway obstruction. International Journal of Pediatric Otorhinolaryngology, 59, 115-118. doi:10.1016/S0165-5876(01)00463-3

[11] Weber, S.A.T., Santos, V.J.B., Semenzati, G.O. and Martim, L.C. (2012) Ambulatory blood pressure monitoring in children with obstructive sleep apnea and primary snoring. International Journal of Pediatric Otorhinolaryngology, 76, 787-790. doi:10.1016/j.ijporl.2012.02.041

[12] Katz, E.S., Greene, M.G., Carson, K.A., Galster, P., Loughlin, G.M., Carroll, L., et al. (2001) Night-to-night variability of polysomnography in children with suspected obstructive sleep apnea. Journal of Pediatrics, 140, 589-594. doi: $10.1067 / \mathrm{mpd} .2002 .123290$

[13] Rimell, F., Rosen, G. and Garcia, J. (1998) Full polysomnographic evaluation of the infant airway. Archives of Otolaryngology-Head and Neck Surgery, 124, 773-776. doi:10.1001/archotol.124.7.773

[14] Nieminen, P., Tolonen, U., Löppönen, H., Löppönen, T., Luotonen, J. and Jokinen, K. (1997) Snoring children: Factors predicting apnea. Acta Oto-Laryngologica, 559, 190-194. doi:10.3109/00016489709124119

[15] Harvey, J.M.M., O’Callaghan, M.J., Wales, P.D., Harris, M.A. and Masters, I.B. (1999) Aetiological factors and development in subjects with obstructive sleep apnea. Journal of Paediatrics and Child Health, 35, 140-144. 
doi:10.1046/j.1440-1754.1999.t01-1-00342.x

[16] Amin, R.S., Kimball, T.R., Bean, J.A., Jeffries, J.L., Willging, J.P., Cotton, R.T., et al. (2002) Left ventricular hypertrophy and abnormal ventricular geometry in children and adolescents with obstructive sleep apnea. American Journal of Respiratory and Critical Care Medicine, 165, 1395-1399. doi:10.1164/rccm.2105118

[17] Kerschner, J.E., Lynch, J.B., Kleiner, H., Flanary, V.A. and Rice, T.B. (2002) Uvulopalatopharyngoplasty with tonsillectomy and adenoidectomy for obstructive sleep apnea in neurologically impaired children. International Journal of Pediatric Otorhinolaryngology, 62, 229-235. doi:10.1016/S0165-5876(01)00623-1

[18] Redline, S., Tishler, P.V., Schluchter, M., Aylor, J., Clark, K. and Graham, G. (1999) Risk factors for sleep-disordered breathing in children: Associations with obesity, race, and respiratory problems. American Journal of Respiratory and Critical Care Medicine, 159, 1527-1532. doi:10.1164/ajrccm.159.5.9809079

[19] Don, D.M., Geller, K.A., Koempel, J.A. and Ward, S.D. (2009) Age specific differences in pediatric obstructive sleep apnea. International Journal of Pediatric Otorhinolaryngology, 73, 1025-1028. doi:10.1016/j.ijporl.2009.04.003

[20] Redline, S., Budhiraja, R., Kapur, V., Marcus, C.L., Mateika, J.H., Mehra, R., Parthasarthy, S., Somers, V.K., Strohl, K.P., Sulit, L.G., Gozal, D., Wise, M.S. and Quan, S.F. (2007) The scoring of respiratory events in sleep: Reliability and validity. Journal of Clinical Sleep Medicine, 3, 169-200.

[21] Ohayon, M.M., Carskadon, M.A., Guilleminault, C. and Vitiello, M.V. (2004) Meta-analysis of quantitative sleep parameters from childhood to old age in healthy individuals: Developing normative sleep values across the human lifespan. Sleep, 27, 1255-1273.

\section{Abbreviations}

AHI: Apnea-Hypopnea Index;

OSAS: Obstructive Sleep Apnea Syndrome;

$\mathrm{mSaO}_{2}$ : mean oxygen saturation;

SBD: Sleep breathing disorder;

SE: Sleep efficiency;

BMI: body mass index. 